Ann. A beille, I962, 5 (3), I8I-I99

\title{
CONTRIBUTION A L'ÉTUDE DE SPHAERULARIA BOMBI (NEMATODA, TYLENCHIDA), PARASITE DES REINES DE BOURDONS
}

\author{
A. POLIRLAC \\ Station de Recherhes sur I Abeille et les Insectes sociunx, Bures-sur-Y'rette (Sine-et-Oise)
}

SOMMAIRE

Les tentatives de domestication des Bourdons, en vue de leur utilisation pour la pollinisation des plantes d'intérêt agricole $s:$ heurtent, en France, à un grave problème de parasitisme.

Le présent mémoire constitue une étude de svonthèsé sur un Nématode. Sphaerularia bombi parasite des reines de Bourdons. duction.

Les principales fonctions organiques du ver sont doninées par une étomnante faculté de repro-

Les effets du parasitisme se traduiraient par une perturbation de la production d'hormone des corpora allata, provorjuée par la décharge de substances toxicuues du parasite. L'inhibition de l'hormone conduit à la castration parasitaire, empêchant les reines de Bourdons d'assurer la pérennité de l'espèce.

\section{INTRODUCTION}

Lors d'un récent symposium international sur la pollinisation, tenu au IJanemark et en Suède (aô̂t I960), l'une des questions les plus importantes a certainement été celle concernant la domestication des Bourdons. L'importance des rapports et le nombre des communications présentés ont fait ressortir la carence des recherches en France, ainsi que le souligne J. LECOMTE dans la conclusion d'un compte rendu ${ }^{(1)}$ de ce symposium : "Le retard de la France en ce domaine paraît indiscutable ; et il est d'autant plus regrettable, qu'étant donné l'importance des facteurs écologiques et éthologiques, il est impossible de se baser sur les recherches étrangères pour résoudre nos problèmes ".

Les spécialistes ont depuis longtemps reconnu l'importance des Bourdons comme agents pollinisateurs de plantes d'intérêt agricole.

On a coutume de considérer l'Abeille domestique (Apis mellifica) comme le plus important et le plus actif de tous les insectes pollinisateurs. Toutefois, si les Bourdons

(1) Annales de l'Abeille, vol. 4, n' ${ }^{0}$,, $196 \mathrm{r}$. 
présentent l'inconvénient de vivre en sociétés moins nombreuses que les Abeilles, ils offrent l'avantage, grâce à la morphologie de leur appareil buccal, de pouvoir procéder plus facilement à la pollinisation de certaines plantes, notamment des 'Trèfles. Certains Trèfles, comme Trifolium repens, ont des nectaires relativement courts et peuvent être aisément fécondés par 1'Abeille, tandis que le Trèfle violet (Trifolium pratense) qui possède une fleur plus longue est spécialement bien fécondé par les Bourdons.

Pour domner une idée de l'importance des Bourdons dans la pollinisation du trèfle violet (Trifolium pratense L.), PEDERsen (I945) a montré que 70 p. Ioo des fleurs pollinisées l'avaient été par des Bourdons, et 30 p. Ioo par les Abeilles. Des observations faites sur la Luzerne (Medicago sativa $L$ ) ont permis de mettre en évidence des différences encore plus nettes; en effet, les Abeilles répugnent à provoquer le " tripping ", mécanisme nécessaire à la fécondation de la Luzerne.

Les colonies de Bourdons peuvent être élevées avec succès en vue de la pollinisation croisée, ainsi que l'ont montré des études de G. NokrgaArd Holm au Danemark (Ig60).

Les problèmes de la pollinisation ne pourront être résolus d'une manière satisfaisante que par des élevages contrôlés de colonies de Bourdons, et une parfaite connaissance de la biologie et de l'écologie des différentes espèces.

Des élevages entrepris (I96I) à la Station de Recherches sur l'Abeille et les Insectes sociaux (Bures-sur-Yvette) avec différentes espèces de Bourdons, ont donné un très faible pourcentage de colonies. Le comportement des reines et l'absence de descendance au cours d'une période tardive (mai-juin) ont révélé, dans la plupart des cas, une atteinte parasitaire par des Nématodes.

L'un des Nématodes parasites d'insectes les plus remarquables est certainement Sphaerularia bombi, forme vivant dans la cavité abdominale des reines de Bourdons. Leur découverte fut l'œuvre de Léon Dufour (I837) chez les deux espèces : Bombus terrestris et Bombus hortulorum.

Après la reconnaissance, par SIEBOLD (I938), de larves typiques de Nématodes, SCHNEIDER (I866) révéla que des appendices, considérés pendant longtemps comme mâles nains, étaient en réalité des gonades femelles hypertrophiées.

LEUCKART (I887) donna une description détaillée de l'anatomie et de l'écologie du parasite.

Une contribution aux connaissances concernant Sphaerularia fut apportée par WACHEK (I955), HATTINGEN (I955) et STEIN (I956).

\section{MÉTHODES EXPÉRIMENTALES ET MATÉRIEL UTILISÉ}

\footnotetext{
Mes recherches ont porté sur des reines de Bourdons élevées en serre, et sur des animaux capturés dans la nature. Le matériel vivant était examiné aussitôt.

Après dissection des reines de Bourdons, les Nématodes sont prélevés et fixés selon la méthode de Looss : les vers sont plongés dans l'alcool à $70^{\circ}$ chauffé jusqu'à une température voisine de $50^{\circ} \mathrm{C}$. Cette température doit permettre une inclusion éventuelle dans la paraffine, en évitant le durcissement des tissus.

Le montage des Nématodes " in toto » en vue d'une détermination a été réalisé selon la technique suivante : les Nématodes sont tués par la chaleur (chauffage pendant quelques secondes à $80^{\circ} \mathrm{C}$ ). Ils sont ensuite fixés durant quelques heures dans une solution de formol acétique (formol à 40 p. Ioo : Io p. Iоo; acide acétique : Io p. IOO). On les transfère ensuite dans du lactophénol d'Amann conte-
} 
nant 0,05 p. Ioo de bleu coton. Ces opérations sont effectuées à chaud, $80^{\circ} \mathrm{C}$ environ, pour éviter les distorsions, pendant quelques secondes. Les spécimens sont alors montés dans du lactophénol contenant une trace de bleu coton; on dispose quelques fragments de laine de verre entre lame et lamelle, pour éviter une compression des Nématodes, susceptible de modifier leurs mensurations.

Les ovaires de reines de Bourdons sont prélevés, aprés dissection de l'abdomen dans le liquide de Ringer, fixés dans l'A. F. A. (') et montés "in toto "après coloration au carmin aluné, ou à l'hémalun-éosine de Mayer.

Des coupes longitudinales d'ovaires nous ont permis une étude histologique plus approfondie; nous avons eu recours à l"inclusion mixte gélose-paraftine, méthode due à cinAtTon.

Des examens de spermathèques ont montré que tuutes les reines prélevées pour expérience étaient fécondées avant d'entrer en hibernation. Ia technirque utiliséte décrite par C. PLATEAuxQuenu (1960), était la suivante:

- On crève la spermathèque sur une lame dans une petite goutte d'eau ; on laisse sécher;

- On verse une goutte de Giemsa R.;

- Au bout d'une minute on ajoute une goutte d'eau;

- Au bout d'une demi-heure on lave par un jet et on observe au microscope.

On peut déjà noter la présence ou l'absence de sperme à la loupe binoculaire. Une spernathèque remplie de sperme apparaît plus réfringente, une spemathèue vierge plus transparente.

\section{DIAGNOSE DU PARASITE}

Sans entrer dans les détails d'une nomenclature trop souvent controversée $\left({ }^{2}\right)$, nous nous bornerons à exposer les caractères particuliers permettant la reconnaissance morphologique et biologique du parasite.

D'après le tableau synoptique des ordres et sous-ordres des Nématodes adultes, comprenant les formes libres et parasites, on relève, parmi les caractères communs des représentants de chaque groupe, les annotations suivantes:

- Absence de glandes caudales et hypodermiques;

- Présence d'un conduit excréteur terminal;

- Organes céphaliques sensoriels papilloïdes ;

- Absence de papilles et de soies somatiques ;

- Mâle pourvu d'une paire de papilles génitales ;

- Glandes œesophagiennes subventrales jamais ouvertes à l'extrémité antérieure de l'cosophage ;

$\rightarrow$ sous-classe : Phasmidia Chitwood et Chitwood, I933.

- Présence d'un stylet;

- Cisophage composé d'un précorpus, d'un métacorpus, d'un isthme et d'une région glandulaire ;

- Cellules intestinales plurinucléées ;

-- Absence de glandes rectales.

$\rightarrow$ ordre : TylENChIDA THORNE, I949.

Thorne: (I949) a rassemblé dans cet ordre les Nématodes munis d'un stylet, par opposition aux Rhabditida, qui en sont dépourvus.

(') L'A. F. A. s'obtient par un mélange des proportions suivantes:

$$
\begin{aligned}
& \text { Alcool } 95^{\circ} \ldots \ldots \ldots \ldots . \\
& \text { Formol } \ldots \ldots \ldots \ldots . \\
& \text { Acide acétique cristal. }
\end{aligned}
$$

(2) VAN ZwaluwenbuRg (I928) avait donné une classification biologique des Nématodes parasites d'Insectes, classification qui ne respectait pas l'ordre systématique et qui avait, de plus, le désavantage de ne pas préciser le cas des espèces qui sont parasites à certains de leurs stades et phorétiques ou commensales à d'autres. 
La glande cosophagienne dorsale s'ouvrant dans le précorpus, près de la basedu stylet, permet d'identifier la super-famille des TýnNchoIdeA, Chitwood et CHITwOod, 1937.

La famille des Allantonematidae ChITwoon et CHrTwood, 1937 présente les. caractères identifiables suivants :

- Stylet fin ;

- Précorpus et Métacorpus sans délimitation nette;

- Musculature œesophagienne peu évidente ;

- Femelle gravide complètement transformée en un "sac reproducteur ";

- Un ovaire ;

- Mâle avec appendices caudaux subterminaux.

FILIPJEV (I929) établit la sous-famille des Sphaerulariinae en considération de Nématodes vivant dans la cavité de certains Insectes.

I. Dufour (I837) créa le genre Sphaerularia pour S. bombi récolté dans la cavité abdominale de reines de Bourdons.

\section{ORGANISATION GÉNÉRALE ET REPRODUC'TION DES PARASITES}

\section{A. - CARACtÈres Morphologiques ét anatomiques des Parasites}

Les larves, les mâles et les femelles juvéniles ont un aspect vermiforme normal, une forme générale cylindrique, avec une partie postérieure effilée et une région antérieure légèrement renflée (fig. I).

Le dimorphisme sexuel est peu prononcé, ainsi qu'en témoignent les mensurations suivantes $\left({ }^{1}\right)$ :

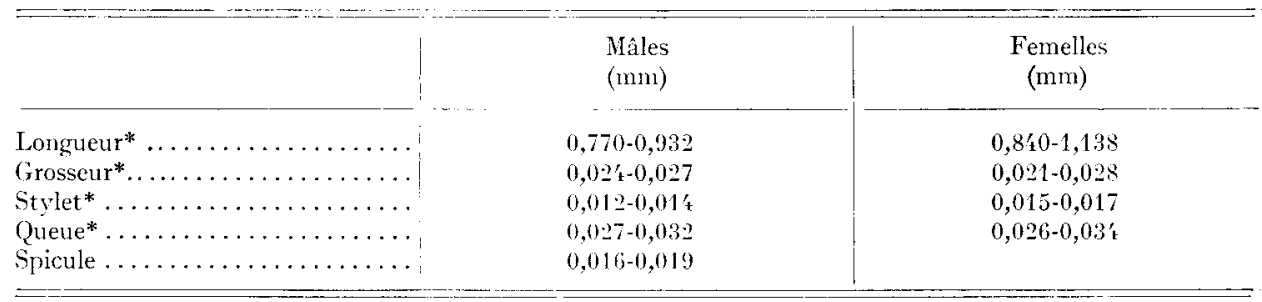

Le corps est revêtu d'une cuticule finement annelée, souple mais inextensible ; cette inextensibilité explique que la croissance s'effectue par mues. Cette annulation est surtout visible dans la région cervicale.

Chez le mâle, un examen superficiel permet de mettre en évidence dans la région caudale, des crêtes longitudinales subterminales permettant le maintien de la femelle pendant la copulation.

Le tube digestif s'étend linéairement dans l'axe du corps entre une bouche terminale et un anus subterminal. Il se compose de quatre éléments principaux :

(1) Les mensurations que j'ai effectuées, marquées d'un astérisque, l'ont été avec une précision allant jusqu'au 1/100 de millimètre. Les autres mesures sont dues à Wacinek.e 


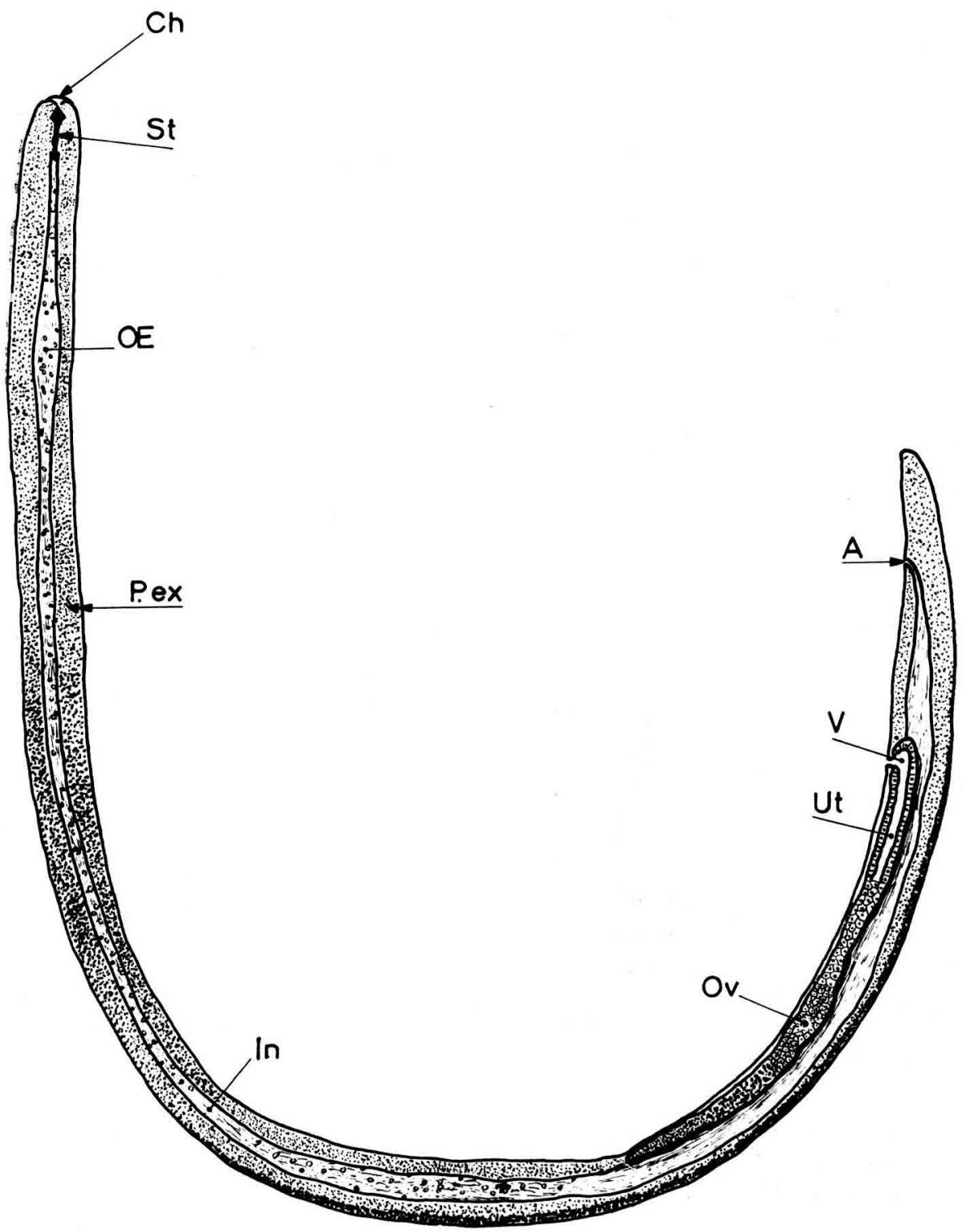

FIG. I. - Sphaerularia bombi : aspect général de la femelle

$\mathrm{A}=$ anus, $\quad \mathrm{Ch}=$ cheilostome,$\quad \mathrm{In}=$ intestin, $\quad(\mathrm{E}=$ esophage, $\quad \mathrm{Ov}=$ ovaire, $\quad$ P.ex. $=$ pore $\mathrm{A}=$ anus, $\mathrm{Ch}=$ cheilostome, $\quad \mathrm{In}=$ intestin,
excréteur, 


\section{a) Bouche et capsule buccale}

La bouche est entourée de lèvres dont la fusion donne à cette région un aspect. uniforme légèrement arrondi (fig. $2 \mathrm{~A}$ ).

A

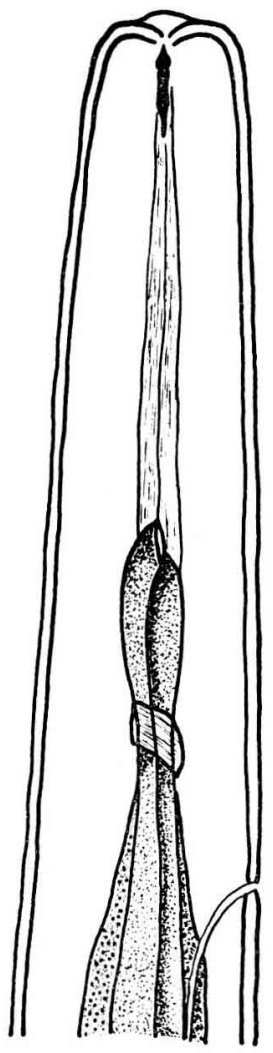

$B$

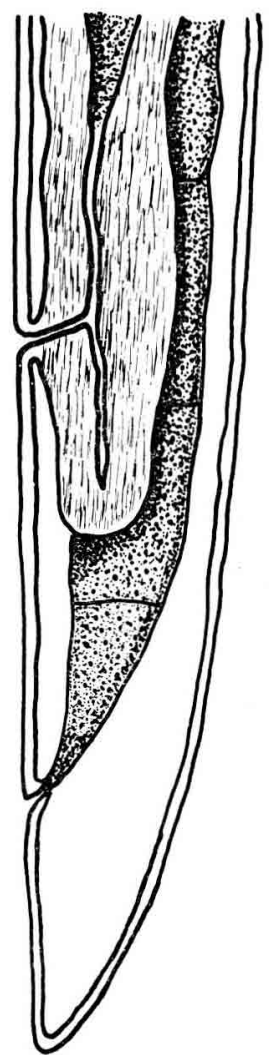

FIG. 2. - Sphaerularia bombi

$\mathrm{A}=$ Région antérieure de la femelle.

$\mathrm{B}=$ Région postérieure de la femelle.

La sclérotisation de la partie antérieure du tube digestif, qui représente probablement le chéilostome, et un stylet buccal (fig. 3), sont les 'seules 'caractéristiques identifiables de cette région.

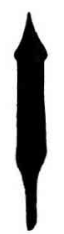

FIG. 3. - Sphaerularia bombi

Stylet buccal. 
Le développement du stylet, dans la famille des Allantonematidae, semble dépendre essentiellement du cycle biologique. Le stylet, bien développé chez les très jeunes larves, subit une dégénérescence partielle - au niveau de la protubérance - dans l'organisme de l'hôte. Le stylet est vestigial chez les mâles adultes, tandis qu'il atteint un grand développement chez les femelles non gravides. Après la copulation, qui s'effectue au cours de la phase libre du cycle des parasites, le mâle meurt, tandis que la femelle, ayant pénétré dans un hôte, laisse voir une dégénérescence du stylet.

\section{b) Esophage}

Cet élément, également appelé pharynx, est constitué d'un précorpus fin, d'un corpus légèrement plus renflé, suivi d'un isthme étroit débouchant dans un bulbe basal bien développé.

Il n'existe pas de valve entre l'œesophage et l'intestin.

Les muscles radiaires de 1'œsophage apparaissent dégénérés, sourtout au niveau de la région bulbaire. Cette dégénérescence de la musculature serait en corrélation avec l'augmentation de volume des glandes œesophagiennes.

Chez les femelles, les glandes cesophagiennes sont nettement reconnaissables au moment de la puberté.

\section{c) Intestin}

L'intestin apparaît comme une masse opaque de globules plus ou moins volumineux, de nature lipidique. Un examen plus approfondi de l'intestin de la femelle nous a permis de constater la présence de globules graisseux dans de grandes cellules peu nombreuses. La couche bacillaire n'a pas été repérable avec les moyens dont nous disposions.

La lumière intestinale est très étroite chez les mâles.

\section{d) Rectum}

La limite de 1'intestin et de la partie terminale du tube digestif est marquée par un petit sphincter.

Les glandes rectales semblent être complètement absentes.

Chez le mâle, les canaux déférents de l'appareil génital débouchent ventralement dans le rectum, encore appelé cloaque, et dont les parois développent divers appendices copulateurs.

Le système alimentaire est peu visible chez les femelles au cours de la reproduction. Cette anomalie est liée à l'énorme développement de l'appareil génital.

\section{B. -- REPRODUCTION DE Sphaerularia bombi}

Les Sphaerularia sont gonochoriques.

Le dimorphisme sexuel est limité aux caractères de l'appareil reproducteur, bien qu'il se manifeste, d'une façon peu nette, par une légère différence de taille, les mâles étant plus courts que les femelles.

L'emplacement des orifices sexuels est différent : les spermiductes débouchent dans la partie terminale du rectum, tandis que l'orifice génital femelle s'ouvre en avant de l'anus et est indépendant du tube digestif (fig. $2 \mathrm{~B}$ ). 
L'extrémité postérieure du corps du mâle est légèrement recourbée ventralement (fig. 4).

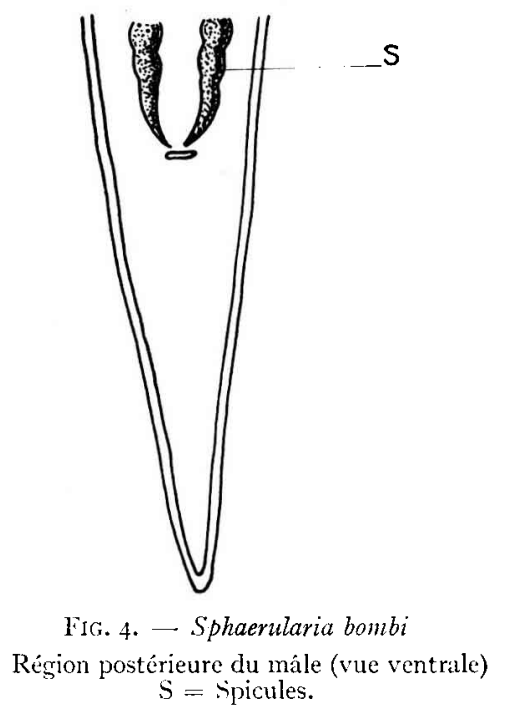

Le mâle possède deux crochets chitineux, les spicules (fig. 5), évaginations de la paroi rectale, qqui servent à maintenir la vulve de la femelle ouverte et joueraient également un rôle dans le cheminement du sperme. Les spicules sont symétriques et ont une forme légèrement ondulée. On note également la présence d'un gubernaculum, plaque cuticulaire, dans les cannelures duquel se déplacent les spicules. Le gubernaculum joue un rôle de guide dans les mouvements des spicules, et de protection pour la paroi de la " poche "spiculaire.

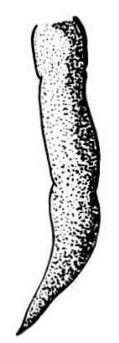

Fig. 5. - Sphaerularia bombi Spicule du mále.

Chez le mâle, il existe un seul testicule, duquel part un canal, le vas efferens qui s'élargit en une vésicule séminale, organe d'accumulation du sperme. Un second canal, le vas deferens, permet l'évacuation du sperme.

Le testicule a une forme allongée, atteignant environ les $2 / 3$ de la longueur du ver.

SEURAT a reconnu que l'appareil reproducteur femelle augmentait en complexité avec le degré de parasitisme. Cette tendance est particulièrement nette dans le groupe des Tylenchoidea. 
Chez la femelle, l'appareil génital ne compte 'qu'un seul ovaire, sorte de sac tubulaire, dans lequel se développent les cellules germinales. Les différents éléments de l'appareil génital femelle (oviducte, utérus, vagin) ne sont différenciés que très difficilement ; seuls la partie terminale du tractus génital et un long sac utérin postvulvaire sont visibles.

Les distances entre l'anus et la vulve varient entre $0,06 \mathrm{Imm}$ et $0,074 \mathrm{~mm}$ : La vulve est localisée à une distance comprise entre I20 et $\mathrm{I} 50$ microns de l'extrémité cauclale.

En période de reproduction, la femelle de Sphaerularia présente des modifications sexuelles considérables. Après la copulation, les femelles pénètrent dans un

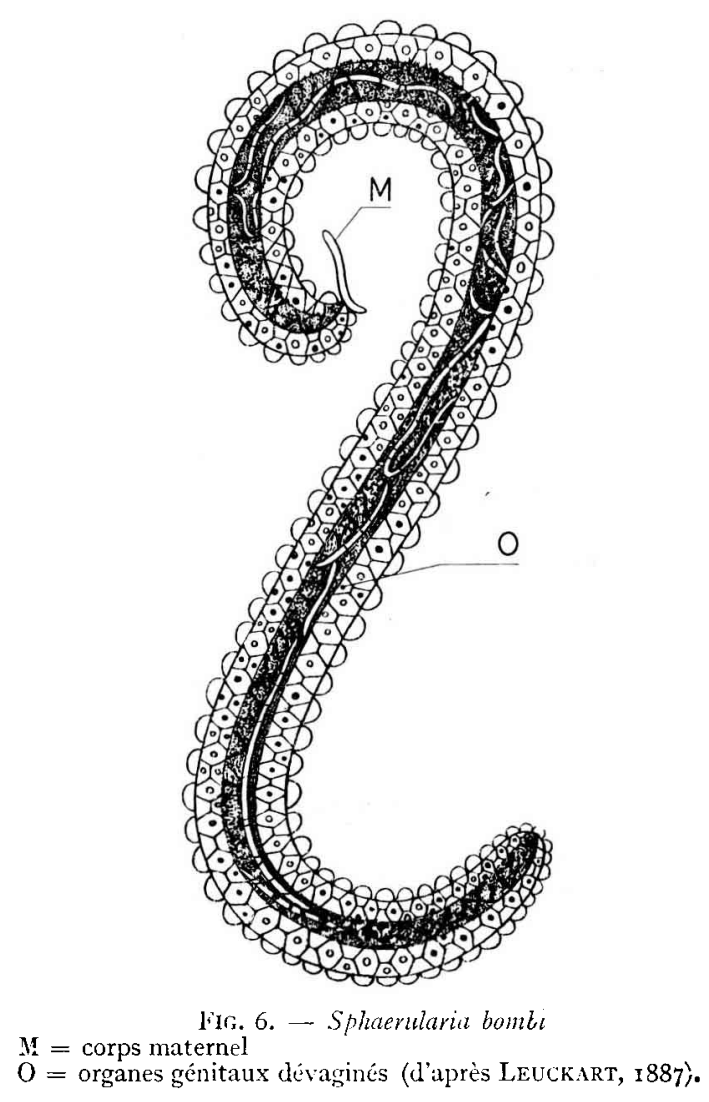

hôte. L'appareil reproducteur du parasite subit alors un accroissement gigantesque, et la gonade se trouve entièrement dévaginée. Il se forme une sorte d'outre, comprenant l'ovaire et l'utérus, et dont la croissance s'effectue par "allongement " des cellules de l'utérus et non par multiplication de celles-ci. Poursuivant son développement à l'intérieur de l'organisme maternel, la gonade hypertrophiée (fig. 6) peut atteindre un volume disproportionné par rapport au corps de la femelle ( $\mathrm{I}_{5}$ ooo à 20 ooo fois plus volumineuse selon certains auteurs). Ein fin de développement, il arrive que le sac utérin se détache du corps maternel. 
Contrairement à la plupart des parasites, il n'y a pas, chez la femelle de Sphaerularia bombi, de mécanismes spécialisés permettant 1'expulsion des cufs, mais le plus souvent il se produit une dégénérescence et même parfois une atrophie de la vulve et du vagin.

\section{DÉVELOPPEMENT DU PARASITE}

Après fécondation dans l'utérus, l'incubation des oufs de Sphaervlaria s'effectue à l'intérieur de "l'outre ", et ceux-ci sont déposés dans le corps de leur hôte.

Le développement embryonnaire a été étudié de façon détaillée par R. HatrisGEN (I955). Tandis que chez les Nématodes on constate généralement un développement eutélique, la segmentation de Sphaerularia bombi présente dès les premiers stades, des irrégularités.

Puisque les œufs de Sphaerularia se développent normalement dans l'hémocoele du Bourdon, on peut supposer que les anomalies observées sont des artefacts, provoqués par des conditions défavorables du développement hors de l'organisme de l'hôte, ou bien qu'ils sont la conséquence d'influences expérimentales externes. Toutefois, ces mêmes formes divergentes ont été observées dans la cavité abdominale de Bourdons fraîchement disséqués. Ia question s'est donc posée de savoir quels étaient les facteurs responsables de variations au cours de la segmentation.

HATTINGEN a supposé que ces modifications étaient provoquées par des influences du milieu : $I^{0}$ pression exercée par l'enveloppe de $1^{\prime} œ u f\left({ }^{1}\right), 2^{0}$ chocs et frottements produits sur l'œuf par les larves déjà écloses.

Des œufs enlevés prématurément de "l'outre ", dépourvus d'enveloppe, présentaient cependant une segmentation normale. L'influence de la membrane de l'œuf n'est donc pas en cause.

L'influence de secousses éprouvées par l'œuf de la part de larves en mouvement a retenu plus longtemps l'attention, sans toutefois fournir une explication satisfaisante. Les cufs subissent des déformations à tel point que la configuration des blastomères s'en trouve modifiée. Des œufs n'ayant subi aucun effet mécanique montrent également des premiers stades de segmentation aberrants.

Ces mécanismes de segmentation anormale seraient dus, en dernière analyse, aux changements de place du fuseau, à la suite de mouvements du cytoplasme.

Le développement postembryonnaire s'effectue par mues successives. Les conditions de survie des larves, extrêmement difficiles à réaliser en conditions expérimentales, ont rendu impossible, jusqu'à présent, la détermination des différents stades larvaires.

Après éclosion, les larves de Sphaerularia bombi ne séjournent que peu de temps dans la cavité abdominale du Bourdon (ro à 20 jours).

Il semble que les larves quittent 1'hôte juste avant la mue imaginale. Ce n'est qu'après la dernière mue qu'est atteinte la maturité génitale. Les stades préadultes sont visibles dans les deux sexes. Les larves femelles montrent les positions de la vulve et du vagin et l'ébauche de l'utérus. Chez les mâles, le testicule est visible, mais aucune évidence des spicules et du gubernaculum.

(') Après la fécondation, l'œuf s'entoure d'une membrane protidique sécrétée par l'épithélium utérin. 
Cycle:

La forme parasite, ou infestante, est constituée par la femelle. Celle-ci pénètre dans 1'hôte-reine de Bourdon en état d'hibernation - après la fertilisation. Les organes génitaux du Nématode ressortent par la vulve, formant un énorme appendice, sorte de sac utérin qui peut se détacher ultérieurement du corps maternel. Les cufs éclosent dans la cavité hémocoelienne de l'hôte, et les larves demeurent là quelque temps. Les larves perforent la paroi intestinale du Bourdon et pénètrent dans la lumière de l'intestin ; elles sont soit éliminées avec les excréments, ou quittent l'hôte par l'anus. HaTTINGEN (I956) a démontré que les larves émigraient du vivant de leur hôte, et non de Bourdons morts.

La forme libre est représentée par les larves, mais nos connaissances sur leur sort sont vagues. Après avoir abandonné la reine de Bourdon, les larves vivent pendant plusieurs mois dans le sol. Certains auteurs (BAYLISS et DAUBNEY) ont supposé que les larves vivaient sans prendre de nourriture, leur bouche étant complètement fermée. A la fin de l'été, les animaux devenus matures s'accouplent. Puis les mâles périssent, tandis que les femelles vont procéder à un nouveau phénomène d'infestation.

\section{RELATIONS ENTRE HOTE E'T PARASITE:}

\section{I. - SPÉCIFICITŚ́ PARASITAIRE}

L'adaptation de Sphaerularia bombi ne concerrie pas exclusivement les reines de Bourdons.

La présence du parasite a été signalée chez les guêpes et les osmies (SchNEIDER, I866 ; STAMMER, I934), mais cette observation n'a jamais été vérifiée positivement.

Nous avons pu constater l'infestation par Sphaerularia parmi toutes les espèces de Bourdons récoltées dans la région parisienne: Bombus terrestris, $B$. lucorum, $B$. lapidarius, B. pratorum, B. hypnorum, B. agrorum; ainsi que chez Psithyrus vestalis et Psithyrus campestris.

Les reines d'Abeilles semblent échapper à cette contamination par Sphaerularia, ce qui s'explique aisément par l'absence de phase terrestre chez Apis.

\section{II. - INTENSITÉ D'INFESTATION}

Les reines de Bourdons infestées le sont avec une intensité variable seion l'année et la saison.

LEUCKART (I887) avait dénombré 32 "outres " de Sphaerularia dans une reine de Bombus terrestris; Hattingen en a compté 34 chez une reine de la même espèce $\left(^{1}\right)$.

(1) Dans le Wisconsin, MEDLer (1957) a montré que ro \% des reines de Bourdons étaient atteintes par Sphaerularia bombi. 
Après dissection d'insectes infestés, j'ai pu constater, en règle générale, la présence d'une seule femelle parasite, parfois deux, mais très rarement trois ou plus (fig. 7).

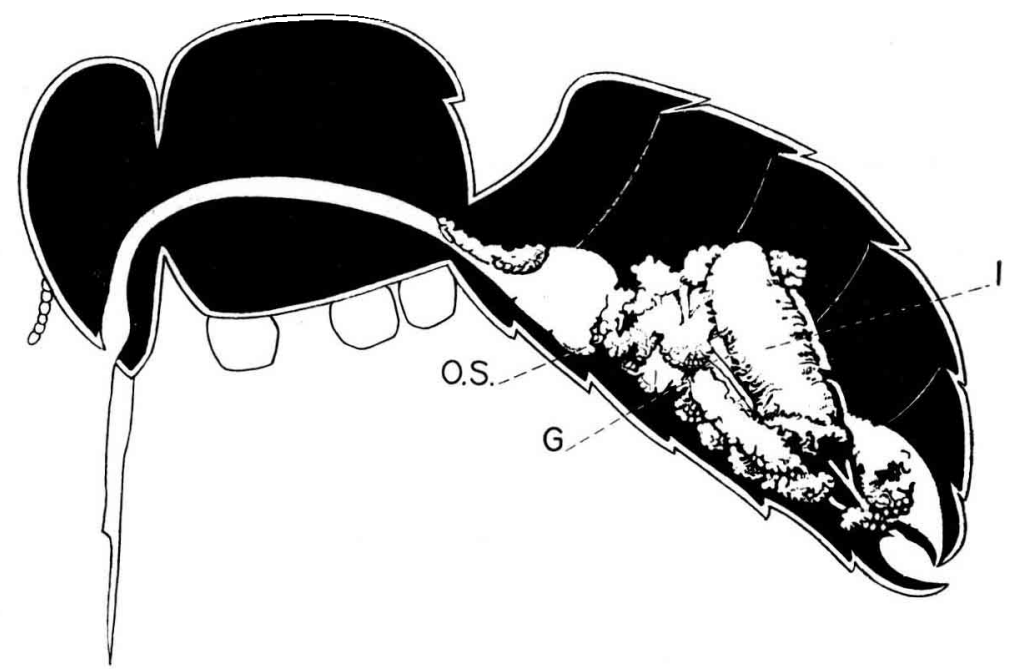

FIG. 7. - Conpe sagitlale d'une reine de Bourdon infestée monirant la position des parasites

$\mathrm{G}=$ Gonade.

$I=$ Intestin

O.S. = "outres" de Sphaenularia (d'aprìs HatTINGEN).

Des observations en serre et dans la nature nous ont permis de constater des modifications importantes du comportement des reines de Bourdons parasitées. Les degrés d'infestation s'accentuent au fur et à mesure du développement des parasites.

Les expériences en serre ont porté essentiellement sur trois espèces de Bourdons. Les résultats sont rapportés dans le tableau suivant:

\section{TABI,EAU I}

Intensite d'infestation en fonction de la période d'examen

\begin{tabular}{|c|c|c|c|c|c|c|}
\hline \multirow{2}{*}{$\begin{array}{c}\text { Périodes } \\
\text { d'examen }(1961)\end{array}$} & \multicolumn{3}{|c|}{ Nombre de reines examinces $\left({ }^{1}\right)$} & \multicolumn{3}{|c|}{$\begin{array}{l}\text { Pourcentage des reines infestées } \\
\text { jar Sphaerularia bombi }\end{array}$} \\
\hline & $\begin{array}{l}\text { B. terres- } \\
\text { tris }\end{array}$ & $\begin{array}{l}\text { B. lapida- } \\
\text { rius }\end{array}$ & $\begin{array}{l}\text { B. agro- } \\
\text { rum }\end{array}$ & B. leneslyis & B. lapidarius & B. agrorum \\
\hline Du 24 avril au 15 mai & 19 & 9 & 16 & $7=37 \%$ & $7=14 \%$ & $7=\ell_{4} \%$ \\
\hline Du 16 mai au 5 juin. & 31 & 11 & 23 & $2 \%=-=77 \%$ & $9=81 \%$ & $19=82 \%$ \\
\hline Du 6 juin au 1 er juil. $^{\text {. }}$ & 22 & 7 & $11_{t}^{\prime}$ & $19=-86 \%$ & $6=85 \%$ & $13=93 \%$ \\
\hline
\end{tabular}

( $\left.{ }^{1}\right)$ Toutes les reines examinćes ont été capturées entre lıur sortiz d'hibernation et l: 24 avril de la même année.

L'examen de ce tableau montre que pour chaque espèce les pourcentages d'infestation sont différemment élevés, ce qui est en rapport avec les modalités d'hibernation. 
L'importance de l'infestation est en relation étroite avec les variations locales ou saisonnières, mais il ne fait aucun doute que cette inhibition des fonctions reproductrices de la reine de Bourdon est d'autant plus marquée qu'elle se situe à une période plus avancée de l'année.

Dès leur sortie d'hibernation (mars-avril), toutes les reines de Bourdons s'affairent avec empressement à la recherche de nourriture, visitant les fleurs pour la récolte du pollen et du nectar. Elles ne manifestent aucun signe extérieur d'atteinte parasitaire. Ce n'est que plus tard, au printemps, que certaines différences peuvent apparaître dans le comportement entre reines saines et reines infestées.

Les reines normales font preuve d'une grande activité dans la recherche et l'aménagement d'un gite, en vue de la fondation d'une colonie. Au cours de cette période les Bourdons infestés survivent mais, affaiblis, ils volent plus lourdement, sans but précis, et montrent peu de vivacité. Ces vols troublés sont caractéristiques, à cette époque tardive, des reines de Bourdons parasitées.

Dans la nature, les Bourdons infestés volent au ras du sol, pénètrent dans un trou, une fissure, ou se dissimulent sous un tas de feuilles. Dans la volière, ils se heurtent aux cloisons vitrées, incapables de diriger leur vol.

En aucun cas, je n'ai observé de nidification par des reines infestées ; toute tentative de procéder à l'élaboration d'un nid semble complètement annihilée.

\section{III. - FACTEURS D'INEESTATION}

Le degré d'infestation des reines de Bourdons par Sphaerularia bombi dépend essentiellement de 3 catégories de facteurs.

\section{a) Facteurs propres au parasite:}

La fécondité de Sphaerularia bombi constitue l'une des caractéristiques les plus importantes dans le domaine du parasitisme. L'hypertrophie des organes génitaux femelles rend compte de la prolifération considérable des parasites, et demeure 1'une des causes de l'intensité d'infestation.

Il n'a pas été possible, jusqu'à présent, de définir avec précision les limites de résistance de Sphaerularia bombi aux températures basses ou élevées. Il ressort des travaux de HATTINGEN (I956) que des températures modérées seraient favorables aux Sphaerularia, tandis que les basses températures les feraient périr. Il existe une relation entre la nature de l'infestation et les conditions météorologiques de la saison froide.

Le fait d'atteindre l'hôte ne constitue qu'une étape de l'infestation ; celle-ci ne peut se maintenir que s'il y a adaptation à l'hôte.

L'éclosion des larves de Sphaerularia s'effectue généralement dans la cavité hémocoelienne de 1'hôte. Toutefois, les parasites peuvent perforer les parois et séjourner dans le tractus intestinal et les gonades, ainsi que nous avons pu le constater.

\section{b) Facteurs propres à l'hôte :}

Les facteurs écologiques et éthologiques sont importants à considérer, car l'infestation ne peut avoir lieu que si l'écologie et l'éthologie du Bourdon lui permettent de rencontrer le parasite. 
A l'automne, les femelles fertilisées de Sphaerularia pénètrent dans la reine de Bourdon au moment où celle-ci, enfouie dans le sol, se trouve en état d'hibernation. Je n'ai jamais trouvé de parasites dans les femelles ouvrières ni dans les mâles de Bourdons ; en effet, ceux-ci disparaissent dès les premiers froids, et ne peuvent hiverner dans la terre.

Certains caractères morphologiques peuvent également favoriser l'infestation. Le mécanisme de pénétration des femelles parasites dans leur hôte n'est pas parfaitement déterminé. ScHNEIDER (I866) supposait que les parasites pénétraient par l'appareil buccal du Bourdon. Il semble qu'il y ait aussi immigration par la membrane intersegmentaire, par le système trachéen et par l'orifice anal. L'abondance de poils et le fait que le segment pygidial ne soit pas hermétiquement fermé constituent des voies de pénétration plus faciles. Il est également possible que les reines soient parasitées au stade larvaire.

\section{IV. - RAPPORTS BIOGÉOGRAPHIQUES}

La modalité de la répartition géographique de Sphaerularia par rapport à celle de son hôte est délicate à établir, car elle nécessite l'examen d'un grand nombre d'exemplaires de Bourdons, provenant de diverses régions de son aire de répartition.

L'infestation a une localisation très étroite. A l'intérieur d'un territoire ( $\left.{ }^{1}\right)$ de capture de Bourdons, situé entre Gif-sur-Yvette et Bures-sur-Yvette et comprenant le Bois des Plants du Moulon, le Chêne vert, le Bois de Courbeville, il a été possible de délimiter deux zones distantes de $\mathrm{L}, 5 \mathrm{~km}$ à $2 \mathrm{~km}$. Dans l'une de ces zones, les reines de Bourdons capturées au printemps ont, soit fondé une colonie, ou n'ont montré aucune atteinte parasitaire par Sphaerularia. Dans l'autre zone, toutes les reines capturées ont témoigné d'une infestation importante.

La cause probable de cette localisation serait le faible rayon d'action des reines de Bourdons. Les possibilités d'infestation d'une aire supposée saine par des Bourdons parasités seraient ainsi limitées. Ces possibilités sont d'autant plus réduites que les deux régions, dépourvue de Sphaerularia et parasitée, sont éloignées l'une de l'autre, ou comportent, du moins, une discontinuité de la flore visitée par les Bourdons.

Il semble donc que le parasite occupe une aire de répartition plus restreinte que celle de son hôte. Peut-être y a-t-il lieu de considérer que les conditions écologiques ne conviennent pas au parasite dans l'ensemble de l'aire de répartition des Bourdons.

\section{V. - ACTIONS DES PARASITES SUR l'ORGANISME DE L'HOTE}

Les premiers symptômes de l'infestation parasitaire se manifestent d'abord sur le comportement des reines. Les reines parasitées continuent de voler jusqu'à une période avancée de l'année (juin-juillet), dû à ce fait qu'elles ne fondent pas de colonies.

(1) Coteaux boisés contenant des feuillus et des Conifères et dont le tapis vécrétal était essentiellement composé de Graminées, de Jacinthes des bois (Indymion nutans, Liliacées) de Pissenlits (Taraxacum Dens leonis, Composées) et de Polygonatum vulgare. 
Aucune nidification n'a été observée par des femelles parasitées. Cette constatation est bien la conséquence de lésions au niveau de l'appareil reproducteur des reines de Bourdons.

Au début de la période d'envol des Bourdons, il n'y a, la plupart du temps, aucune différence morphologique entre les gonades des reines saines et celles des reines infestées. Le Nématode n'exercerait donc pas de préjudices sur l'organisme de son hôte au cours du repos hivernal.

PALM (I94S) et HAtTIXGEx ( 1956$)$ ont remarqué que non seulement les ovocytes et les cellules nourricières, mais également la chambre terminale de lovariole de femelles parasitées, étaient prodondément modifiées.

In examen histologique montre non seulement un arrêt du développement ovarien, mais encore une dégénérescence des divers éléments de l'ovaire. La traumatisation affecte d'abord les ovocytes et les cellules nourricières. L'ovocyte dégénére et se troure résorbé par l'épithélium folliculaire qui l'entoure; cet épithélium se transforme alors en une structure assez semblable à celle du corpus luteum de l'ovaire normal. Plus tarł, les cellules nourricières se désagrègent également et sont résorbées. Dans quelques cas, les troubles du développement ovocytaire peuvent ne se manifester qu'assez tard, c'est-à-dire après la formation du chorion.

I es dissections de reines parasitées m'ont permis d'observer l'état de développement ovarien, en fonction de la période où les insectes ont péri.

Les résultats du tableau 2 corroborent ceux du tableau I ; autrement dit 1'inhibition des fonctions reproductrices, d'autant plus accentuée que la saison est plus avancée, est en corrélation étroite avec l'action traumatique provoquée au niveau des ovaires.

Ovocytes et cellules nourricières montrent une dégénérescence progressive qui s'achève par la liquéfaction du vitellus. La chambre terminale de l'ovariole ne produit plus d'ovocytes. Les ovocytes dégénérés ne sont pas remplacés. La résorption de la masse vitelline donne alors aux gonades un aspect lacunaire caractéristique. Les chambres d'orocỹtes vides constituent le stade final de la dégénérescence; elles contiennent parfois encore quelques particules incohérentes - fragments de chromatine.

Les modifications dues au parasitisme ne sont pas restreintes à la castration parasitaire. Un examen attentif des corpora allata a montré que ceux-ci subissaient aussi un arrêt de développement; des différences parfois importantes apparaissent entre les glandes des reines parasitées et celles des reines saines, les corpora allata des animaux infestés se trouvant réduits. Les modifications histologiques ne sont pas toujours très distinctes.

La véritable nature des effets causés par le parasitisme s'avère complexe. On considère que les effets sont généralement dus à une privation de nourriture de l'hôte, provoqués par la suppression de quantités considérables de substances nécessaires. Cependant, l'inanition est d'une importance minime dans le cas de l'infestation par Sphaerularia.

Le ver serait un véritable organisme pathogène, déchargeant une substance toxique qui est responsable de la plupart des troubles chez les femelles parasitées. Palur en a donné les raisons suivantes

- un examen du corps adipeux de reines infestées et de reines saines a montré qu'aucune différence appréciable n'intervenait dans le volume et la teneur du corps adipeux ; 
TABLEAU 2

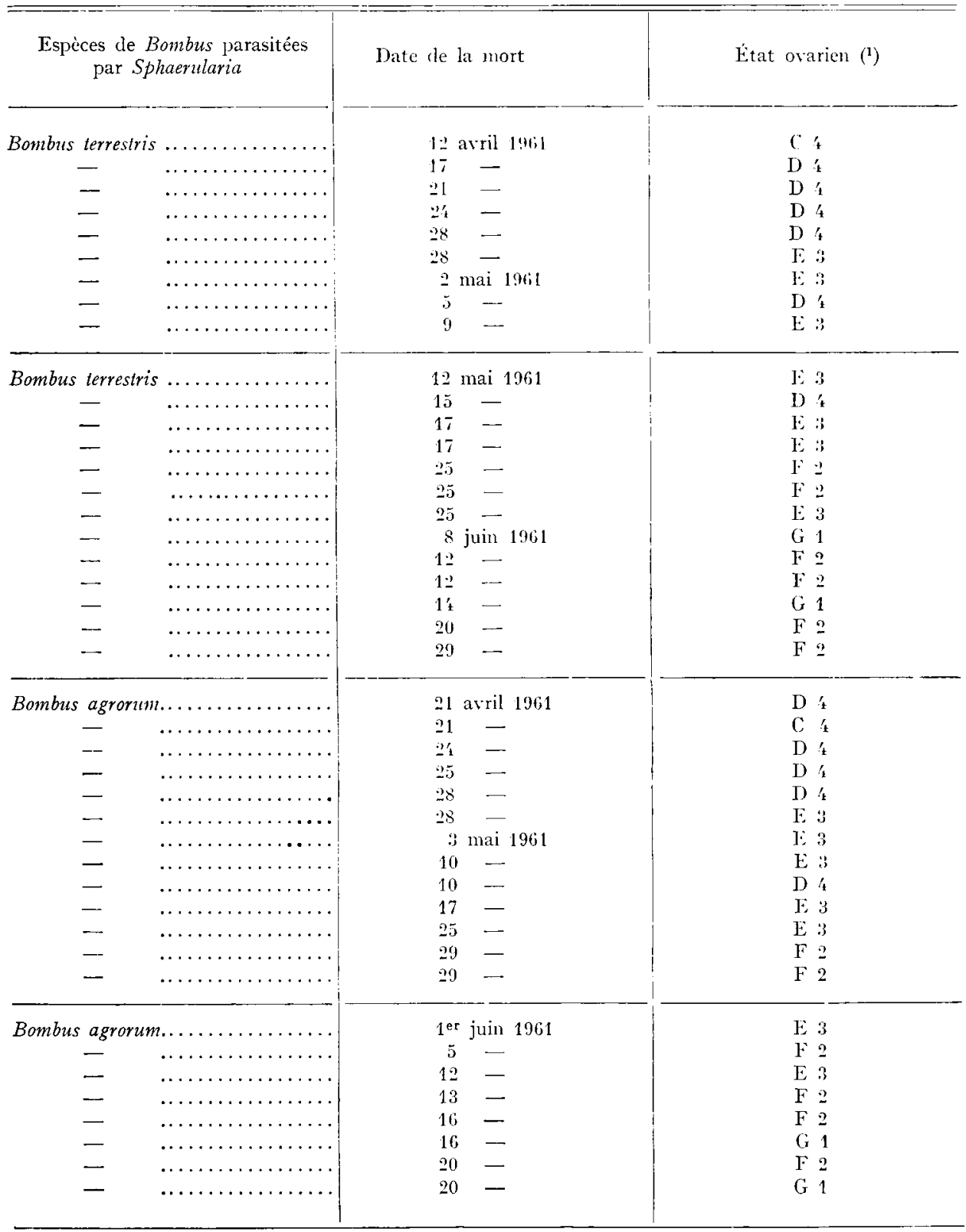

( ${ }^{1}$ Lettres et chiffres correspondent à ceux attribués par G. HEss ( Ueber den Einfuss der Wisellosigkeit und des Fruchtbarkeitsvitamins E auf die Ovarien der Bienenarbeiterin * I942. p. 54) pour caractériser l'état de développement ovarien d'Apis mellifica (fig. 8). 


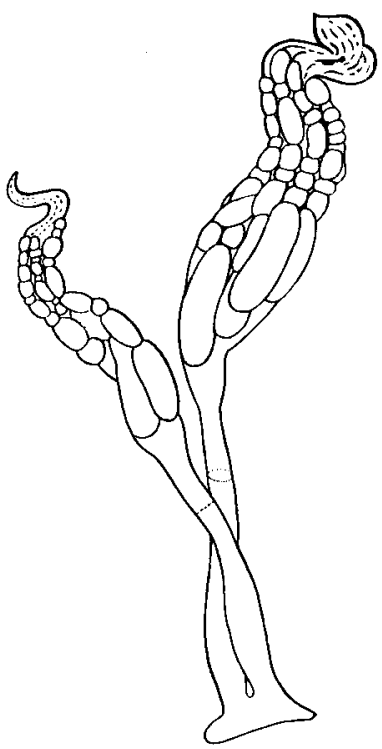

A

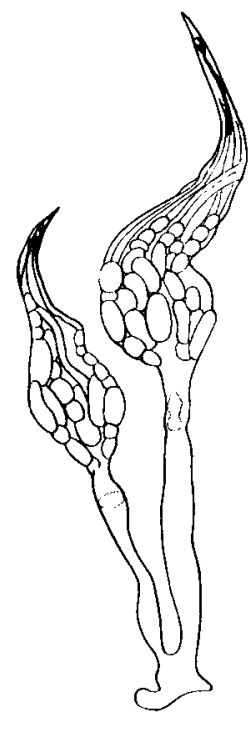

$\mathrm{B}$

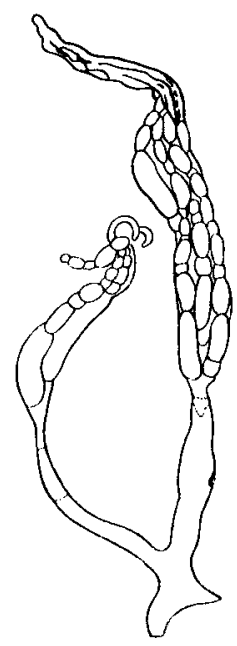

C
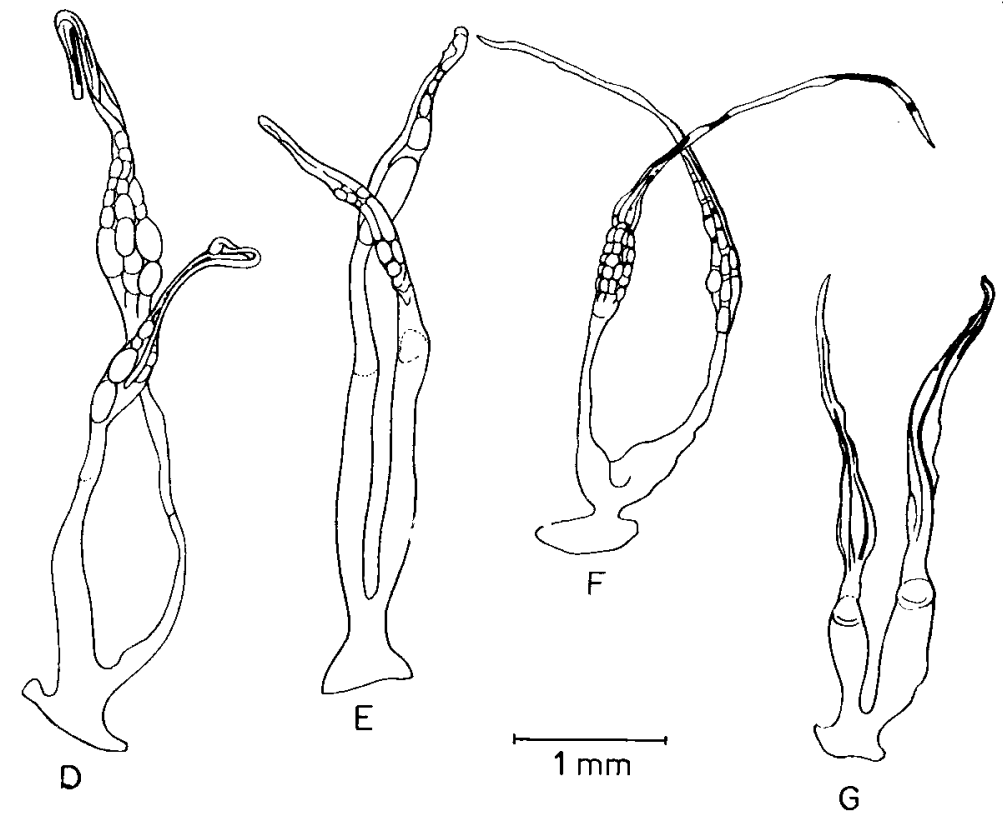

FIG. 8. - Tableau montrant le développement ovarien (d'après G. Hess).

- les troubles provoqués sont indépendants du nombre de parasites présents dans la cavité de l'hôte ;

- la croissance de l'appendice génital du parasite a lieu pendant l'hiver, mais 
les ovaires ne commencent leur croissance qu'après la sortie au printemps. Pendant cette période, les Bourdons ne semblent pas avoir de difficultés dans l'accumulation de leur nourriture.

Bien qu'aucune connexion entre les corpora allata et les organes reproducteurs n'ait été démontrée expérimentalement chez les Apides, il n'y a aucune raison de douter de son existence.

Pendant l'hiver, les cellules épithéliales des corpora allata des reines s'accroissent normalement, et produisent une hormone gonadotrope qui favorise la croissance des ovocytes et des structures annexes des ovaires.

L'interruption de la croissance des corpora allata chez les Bourdons infestés par Sphaerularia s'est déjà produite avant la réapparition de la reine au printemps ; c'est pourquoi on peut conclure que cette insuffisance des corpora allata est en rapport avec le manque de développement des ovaires. Ces insectes présenteraient une insuffisance d'hormone gonadotrope, qui est la cause directe de l'arrêt du développement ovarien conduisant à la castration parasitaire, tandis que les perturbations des corpora allata constitueraient les effets essentiels du parasitisme.

\section{CONCLUSION}

Parmi les Nématodes parasites d'insectes, le genre Sphaerularia est digne d'attention à cause des caractéristiques particulières de la femelle, de laquelle les organes reproducteurs fertilisés sont complètement ressortis. L'utérus gravide se développe considérablement et le reste du corps diminue jusqu'à ce qu'il n'apparaisse plus que comme un appendice de l'utérus hypertrophié. Le Nématode, non seulement prélève sa nourriture aux dépens de l'hôte, mais aussi constitue un obstacle à la croissance et aux fonctions organiques primordiales de la reine de Bourdon.

Sphaerularia joue un rôle économique important. Sa présence chez les reines de Bourdons inhibe la croissance des ovaires, empêche la ponte et la fondation de nouvelles colonies. Ceci a pour résultat de réduire les populations de Bourdons, nécessaires à la pollinisation de plantes d'un intérêt agricole extrêmement important.

Si la domestication des Bourdons nécessite une connaissance complète de leur biologie, certaines conditions peuvent être réalisées en vue d'une utilisation pratique, notamment la capture des reines en début de saison, dès leur sortie d'hibernation, ce qui éliminerait en majeure partie, les risques d'obtenir des reines de Bourdons parasitées par Sphaerularia bombi.

Reçu pour publication en mai 1962

\section{SUMMARY}

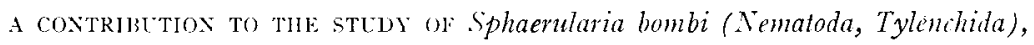
A PARASITE OF BOMBLS QLEENS

No study of synthesis had been given to nematoda Sphaerularia bombi.

At the beginning of this work, we expose the used experimental methods for the cletermination, and the anatomic and biological studies of the parasite, as well as the technics of taking and study of ovaries and appendages of Bombus queen. 
After we had recalled and specified the systematic position of Sphaerularia bombi, we have undertaken a study of the morphology and of the main organic functions of the worm, dominated, in fact, by a wonderful property of reproduction.

Then the development and life cycle of Sphaerularia bombi are looked at.

Finally, we have considered the relations between the parasites and their host; the specificity and the intensity of infestation, particularly for B. terrestris, lapidarius and agrorum.

The biogeographic relations show that the parasite has a more restricted distribution than that of its host.

The effects of the parasite on Bombus queens result in a disturbance in the hormone production from the corpora allata, caused by the discharge of toxic products by the parasite. The lack of corpora allata hormone causes the parasitic castration, and this is a secondary effect of the paras itation.

\section{RÉFÉRENCES BIBLIOGRAPHIQUES}

- Chiswood B. G., Cintwood M. B., 1950. An introduction to nematology. Section I, Anatomy. Monumental Printing Company, Baltimore.

Dufotir L., 1837. Recherches sur quelques entozoaires et larves parasites des Insectes orthoptères et hyménoptères. Ann. Sci. Nat. Zool., 1, 5-20.

Filipjev I. N., Schudrmans Stekhoven Jr. I94I. A manual of agricultural helminthology. E. J. Brill Leiden, Holland.

Free J. B., BUTler C. C., 1959. Bumblebees. The Iac Millan company, New-York, 208 p.

Hattingen R., 1956. Beiträge zur Biologie von Splacerularia bombi-Léon Dufour (1837). Zentralbl.t. . Bakteriologie, Parasitenkunde, Infekt. u. Hygiene, II Abt., 109, 236-249.

HATTINGEN R., I956. Beiträge zur Entwicklungsgeschichte von Sphaerularia bombi - L. Dufour (I837). Roux'Arch.f. Entw., 148, 494-503.

Holm S. N., Hass H., I960. Humlebierne, deres betydning og nulighederne for deres domesticering. Soertryk af Tolvmandsbladet, $\mathrm{n}^{\circ} 8$.

LEUCKART R., r887. Neue Beiträge zur Kenntnis des Baues und der Lebensgeschichte der Nomatoden. Abh. kgl.-sachs. Ges. Wiss., 22, 567-704.

Palm N.B., I 948. Normal and pathological histology of the ovaries in Bombus Latr. (Hymenopt.) Opuscula Entomologica Supplemenium, 7, 101 p.

Pebersen A., 1945. Rodkloverens blomstring og bestovning. Den kgl. Veterinaer-og Land-bohojskoles Arsskritt.

Schneider A., i885. Ueber die Entwicklung der Sphaerularia bombi. Zool. Beitr. v. Schneider, 1.

Sellier R., i959. Jes Insectes utiles. Payot, Paris, 286 p.

Selrat L. G., igr6. Contribution à l'étude des formes larvaires des Nématodes hétéroxènes. Bull. Sci. Fr. Belg., 49, 297-377.

STEIN G., I 956. Weitere Beiträge zur Biologie von Sphaerularia bombi. — Léon Dufour (1837). Z.f. Parasitenkunde, 1\%, 383-393.

Van Zwaluwendurg R. H, 1928. The interrelationships of insects and roundworms. Bull. Exp. Sta. Haw. Sug. Plant., Ent. Ser., 20, 68 p.

Wachek F., I955. Die entoparasitischen Tylenchiden. Parasit. Schrijt. 3, i 9 p. 